ORIGINAL ARTICLE

\title{
AP2 $\gamma$ controls adult hippocampal neurogenesis and modulates cognitive, but not anxiety or depressive-like behavior
}

\author{
A Mateus-Pinheiro ${ }^{1,2,6}$, ND Alves ${ }^{1,2,6}$, P Patrício $^{1,2}$, AR Machado-Santos $^{1,2}$, E Loureiro-Campos $^{1,2}$, JM Silva ${ }^{1,2}$, VM Sardinha ${ }^{1,2}$, J Reis $^{1,2}$, \\ H Schorle $^{3}$, JF Oliveira ${ }^{1,2}$, J Ninkovic ${ }^{4,5}$, N Sousa $^{1,2}$ and L Pinto ${ }^{1,2}$
}

Hippocampal neurogenesis has been proposed to participate in a myriad of behavioral responses, both in basal states and in the context of neuropsychiatric disorders. Here, we identify activating protein $2 \gamma$ (AP2 $\gamma$, also known as Tcfap2c), originally described to regulate the generation of neurons in the developing cortex, as a modulator of adult hippocampal glutamatergic neurogenesis in mice. Specifically, AP2 $\gamma$ is present in a sub-population of hippocampal transient amplifying progenitors. There, it is found to act as a positive regulator of the cell fate determinants Tbr2 and NeuroD, promoting proliferation and differentiation of new glutamatergic granular neurons. Conditional ablation of $\mathrm{AP} 2 \gamma$ in the adult brain significantly reduced hippocampal neurogenesis and disrupted neural coherence between the ventral hippocampus and the medial prefrontal cortex. Furthermore, it resulted in the precipitation of multimodal cognitive deficits. This indicates that the sub-population of AP2 $\gamma$-positive hippocampal progenitors may constitute an important cellular substrate for hippocampal-dependent cognitive functions. Concurrently, AP2 $\gamma$ deletion produced significant impairments in contextual memory and reversal learning. More so, in a water maze reference memory task a delay in the transition to cognitive strategies relying on hippocampal function integrity was observed. Interestingly, anxiety- and depressive-like behaviors were not significantly affected. Altogether, findings open new perspectives in understanding the role of specific sub-populations of newborn neurons in the (patho)physiology of neuropsychiatric disorders affecting hippocampal neuroplasticity and cognitive function in the adult brain.

Molecular Psychiatry advance online publication, 25 October 2016; doi:10.1038/mp.2016.169

\section{INTRODUCTION}

In the adult central nervous system, specific brain niches retain the ability to generate new neurons throughout life. ${ }^{1}$ Among these, the subgranular zone (SGZ) of the hippocampal dentate gyrus (DG) is of particular interest. There, newly generated cells become mostly glutamatergic granular neurons, ${ }^{2-4}$ in the process recognized as neurogenesis. Adult hippocampal neurogenesis is a multistep and highly regulated process, originating from neural stem cells (NSCs) residing in the SGZ. ${ }^{1,5}$ Thereafter, the SGZ NSCs will divide to give rise to transient amplifying progenitors (TAPs), mitotically active cells, which will be responsible for the rapid expansion of the multipotent progenitor cells pool. Finally, the generated neuroblasts will undergo a short migration into the granule cell layer of the DG, differentiating into fully mature and integrated neurons in the pre-existing neural circuits. Importantly, survival of newborn cells depends on proper axonal and dendritic development. This confers cells the ability to receive GABAergic and, subsequently, glutamatergic synaptic input, both crucial for normal maturation and integration of newly generated cells. ${ }^{6}$

Several lines of evidence have shed light on the relevance of hippocampal neurogenesis for both structural and functional plasticity of the adult hippocampus. This process has behavioral repercussions in distinct cognitive and emotional domains, both in basal states and in neuropsychiatric disorders (such as schizophrenia and depressive disorders). ${ }^{7-11}$ More so, the transcriptional network involved in the regulation of neurogenesis, both in early developmental stages and during adulthood, has been the focus of recent studies. ${ }^{12-16}$ It is now established that during cortical development the regulation of glutamatergic neurogenesis is controlled by a set of transcription factors, including Pax6, Tbr2, NeuroD and Tbr1, with implications on proliferation, cell cycle kinetics, lineage and fate specification, axonal growth and cell adhesion processes. ${ }^{13,17,18}$ Interestingly, the transcriptional sequence of cell fate determinants (Pax $6 \rightarrow$ Tbr2 $\rightarrow$ NeuroD $\rightarrow$ Tbr 1 ) is recapitulated during adult hippocampal neurogenesis and, with some variations, has a role in cell fate towards glutamatergic lineages in the subependymal zone. ${ }^{16,18-22}$

Activating protein $2 \gamma$ (AP2 $\gamma$, also known as Tcfap2c or Tfap2c) is a recently described transcription factor. It is part of the transcriptional network regulating glutamatergic neurogenesis during early developmental stages, directly regulating the basal progenitor fate determinants Math3 and Tbr2. In the developing cortex, deletion of $\mathrm{AP} 2 \gamma$ results in a specific reduction of upper layer neurons in the occipital cerebral cortex, whereas its overexpression potentiates region- and time-specific generation of cortical layers II/III. ${ }^{23}$ Yet, during adulthood, AP2 $\gamma$ has been classically linked to breast carcinogenesis, namely as a promotor of proliferation and impaired differentiation of tumor cells and as

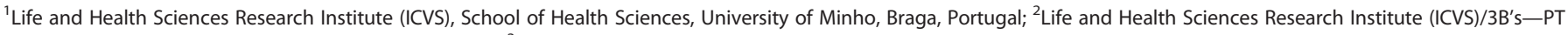
Government Associate Laboratory, Guimarães, Portugal; ${ }^{3}$ Department of Developmental Pathology, Institute for Pathology, University of Bonn Medical School, Bonn, Germany; ${ }^{4}$ Institute for Stem Cell Research, Helmholtz Centre Munich German Research Center for Environmental Health (GmbH), Neuherberg, Germany and ${ }^{5}$ Physiological Genomics,

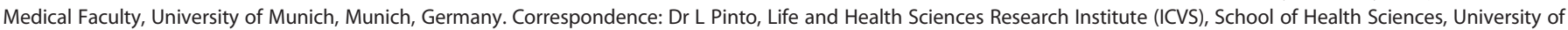
Minho, Campus de Gualtar, Braga 4710-057, Portugal.

E-mail: luisapinto@ecsaude.uminho.pt

${ }^{6}$ These authors contributed equally to this work.

Received 21 June 2016; revised 27 July 2016; accepted 4 August 2016
} 
a contributor to chemoresistance and radiation resistance of these cells. ${ }^{24}$

Herein, in a mice model, we addressed the question of whether AP2 $\gamma$ is an active transcriptional regulator of adult glutamatergic neurogenesis and if its function is relevant for different emotional and cognitive behavioral dimensions. The present study reveals an important role of $\mathrm{AP} 2 \gamma$ in the regulation of glutamatergic neurogenesis in the adult hippocampal DG, with functional repercussions in the integrity of limbicocortical connections and in different cognitive modalities.

\section{MATERIALS AND METHODS}

A brief description of the Materials and methods is presented in this section. For a full description of all methods, please refer to the Supplementary Information.

\section{Animals}

AP2 $\gamma^{\text {loxp/loxp }}$ (AP2yfl/fl), Emx1-cre, Glast:CreErt2 (ref. 25) and Glast:CreErt2/Z/ $E G^{26}$ mice were maintained on a C57BI/6J background (also used as wild type). For the initial in vivo AP2 $\gamma$ deletion experiment, AP2 $\gamma f l / f$ mice were crossed with Glast:CreErt2/Z/EG mice to generate AP2yfl/fl//Glast::CreErt2//Z/ EG mice. Tamoxifen (Sigma-Aldrich, St Louis, MO, USA; T-5648) was dissolved in corn oil (Sigma-Aldrich; C-8267) at $20 \mathrm{mg} \mathrm{ml}^{-1}$ and $1 \mathrm{mg}$ was injected intraperitoneally two times a day for 5 consecutive days in 2-month-old male animals. Animals were killed 1 week after the end of tamoxifen administration. For in vivo AP2 $\gamma$ overexpression experiments, 2-month-old male C57BI/6J wild-type animals were stereotactically injected with $1 \mu \mathrm{l}$ of either CAG-IRES-GFP (IRES-GFP) or CAG-IRES-AP2 $\gamma$ (AP2 $\gamma$-IRES-GFP) retroviruses into the left and the right DG, and killed either 1 week or 1 month postinjections ( $n=5$ per group for each experimental condition). For behavioral and electrophysiological studies, wild-type (Wt), AP2 $\gamma f l /+/ /$ Glast::CreErt2 (AP2 $\gamma^{+/-}$CKO) and AP2 $\gamma f l / f l / / G l a s t:: C r e E r t 2 ~\left(A P 2 \gamma^{-\prime}-\right.$ cKO) 2-month-old male mice were injected intraperitoneally with $1 \mathrm{mg}$ tamoxifen two times a day for 5 consecutive days, with 7 days break followed by injections for 5 additional consecutive days. Animals were subjected to electrophysiological studies and behavioral testing 21 days after injections ( $n=10$ per group).

All procedures were carried out in accordance with EU Directive 2010/63/EU and were approved by the Portuguese Government/Direção Geral de Alimentação e Veterinária (DGAV) with the project reference 0420/000/000/2011 (DGAV 4542).

\section{In situ hybridization and immunohistochemical analysis}

In situ hybridization and immunostaining analysis were performed as described previously. ${ }^{23}$ Details on conditions and antibodies can be found in the Supplementary Information.

\section{BrdU labeling}

Wt mice used for cell type analyses with in situ hybridization and immunofluorescence were given bromodeoxyuridine (BrdU) in drinking water ( $1 \mathrm{mg} \mathrm{ml}^{-1}$; Sigma-Aldrich; B5002) for 2 weeks, and killed 8 weeks later. For the remaining deletion and overexpression experiments, mice were injected once with BrdU (100 $\mathrm{mg} \mathrm{kg}^{-1}$, intraperitoneally), $24 \mathrm{~h}$ before killing.

\section{Primary DG cultures and in vitro AP2 $\gamma$ deletion}

For primary DG cultures, six male mice (AP2yfl/fl male mice, 2 months old) were used, as described previously. ${ }^{23}$ Cells were transduced with a retroviral vector IRES-GFP or CRE-IRES-GFP $2 \mathrm{~h}$ after being plated. ${ }^{27}$ After 7 days in culture, cells were fixed with $4 \%$ paraformaldehyde in PBS for $15 \mathrm{~min}$. at room temperature and processed for antibody staining.

\section{D morphological analysis}

To assess the 3D dendritic morphology of hippocampal DG granular neurons, we used the Golgi-Cox impregnation technique. Dendritic arborization and spine numbers/density were analyzed in the DG of $\mathrm{Wt}, \mathrm{AP}_{2} \gamma^{+/-} \mathrm{CKO}$ and $\mathrm{AP} 2 \gamma^{-1-} \mathrm{CKO}$ mice, as described previously ${ }^{7,9}$ (10-15 neurons for each animal; $n=4$ per group).
Electrophysiological studies

Local field potentials (LFPs) were recorded in the ventral hippocampus (vHIP) and in the prefrontal cortex (PFC); coherence measurements between simultaneously recorded LFPs in both regions were performed, as described previously. ${ }^{28}$ Power spectra densities (PSDs) were also measured in these two regions, as detailed in the Supplementary Information.

\section{Behavioral analysis}

$\mathrm{Wt}, \mathrm{AP} 2 \mathrm{\gamma}^{+/-}$CKO and $\mathrm{AP} 2 \mathrm{Y}^{-1-}$ CKO mice were tested in the forced swimming test (FST; to assess depressive-like behavior), in the open field and in the elevated plus maze tests (to assess anxiety-like behavior), as described previously. ${ }^{9}$ Furthermore, mice were tested in a contextual fear conditioning paradigm, as well as in different water maze tasks to characterize animals' cognitive function, as detailed in the Supplementary Information.

\section{Data analysis and statistics}

Statistical analyses were performed using the SPSS software (Chicago, IL, USA). Animals were assigned to groups according to their genotypes. Sample sizes were determined by power analyses based on previously published studies. All presented data satisfied normal distribution in Kolmogorov-Smirnov testing. After confirmation of homogeneity of group variances between the groups, data were subjected to appropriate statistical tests. Analysis of variance (ANOVA) repeated measures was used to analyze performance on cognitive learning tasks. One-way ANOVA was used to evaluate the remaining behavioral and molecular results. $F$ - and $P$ values derived from statistical analyses are properly indicated along the text. Differences between groups were determined by Bonferroni's post hoc multiple comparison test, and the corresponding $P$-values are indicated in the figures. A $t$-test was used to evaluate differences between two groups where appropriate. Statistical significance was accepted for $P<0.05$. No data points were excluded from the different analyses. Effect size, Cohen's $d$ for $t$-test and $\eta^{2}$ for ANOVA were presented whenever statistical significance was reached. All results and corresponding statistical analyses are detailed in Supplementary Table 1.

\section{RESULTS}

$\mathrm{AP} 2 \gamma$ is present in the adult hippocampal neurogenic niche In light of the early description of the role of AP2 $\gamma$ in the regulation of glutamatergic neurogenesis during developmental stages, we explored whether AP2 $\gamma$ expression was present in the adult hippocampal DG, as this area represents an important source of glutamatergic neurons in the adult brain. Using in situ hybridization to characterize regional gene expression distribution, we found AP2 $\gamma$-mRNA-positive cells in the adult DG (Figure 1a). Furthermore, using an 8-week BrdU label retaining protocol, we found colocalization of AP2 $\gamma$-mRNA signal with BrdU labeling, as well as with the transcription factor Tbr2, a regulator of glutamatergic neurogenesis in both developing and mature brain (Figure 1b). Subsequent immunofluorescent labeling of AP2 $\gamma$ protein and cell count analysis revealed a high proportion of AP2 $\gamma$-positive cells in the SGZ to be also positive for the neuroblast marker doublecortin (DCX) $(61.5 \pm 2.7 \%)$, whereas a subset of these cells was colabelled with Tbr2 $(21.3 \pm 4.1 \%$; Figures $1 \mathrm{c}$ and $\mathrm{d}$ ), supporting lineage commitment of AP2 $\gamma$-positive cells to the glutamatergic neuronal lineage. Moreover, AP2 $\gamma$ immunopositive cells were also positive for the cell cycle marker Ki-67 (Supplementary Figure 1) and BrdU (after an 8week chase period) in the hippocampal DG $(13.9 \pm 3.5 \%$; Figures $1 \mathrm{c}$ and $\mathrm{d}$ ), showing that a small portion of AP2 $\gamma$-positive cells are slow dividing progenitor cells. Importantly, we did not find colocalization between AP2 $\gamma$-positive cells and mature neuronal nuclei (NeuN)-positive neurons (Supplementary Figure 1). 
a

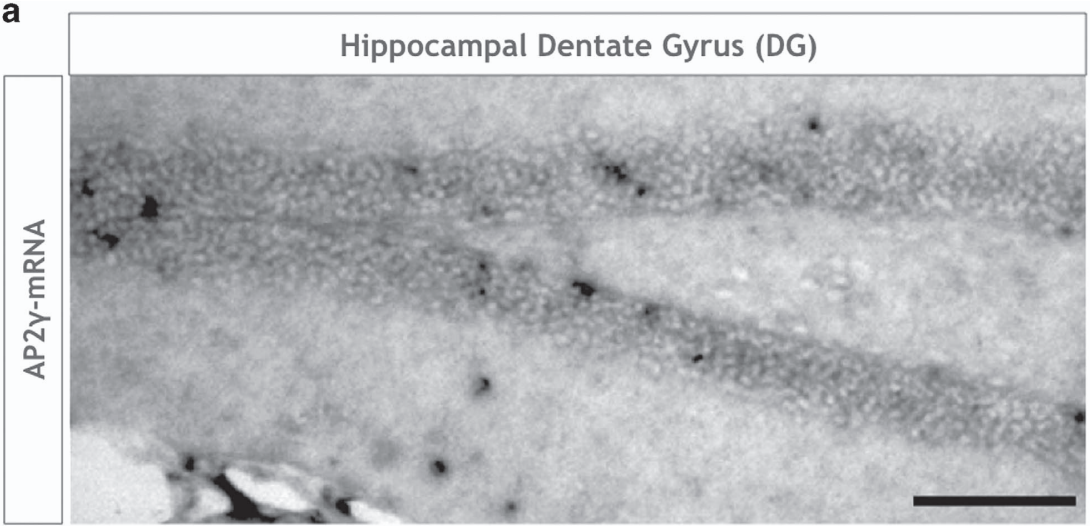

b
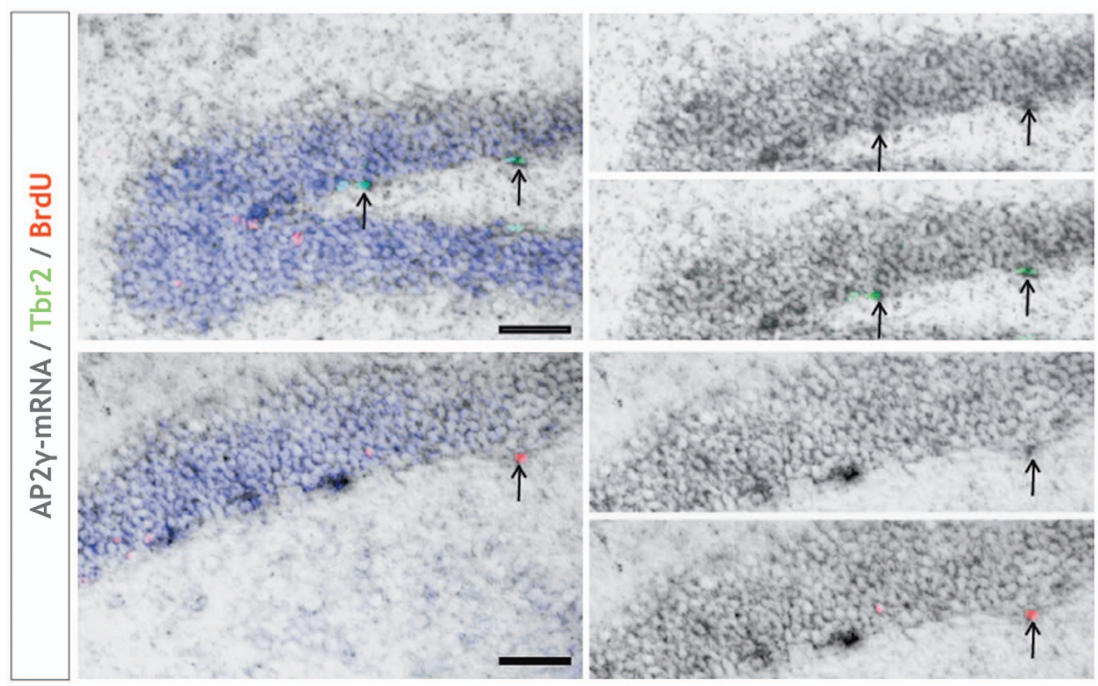

C

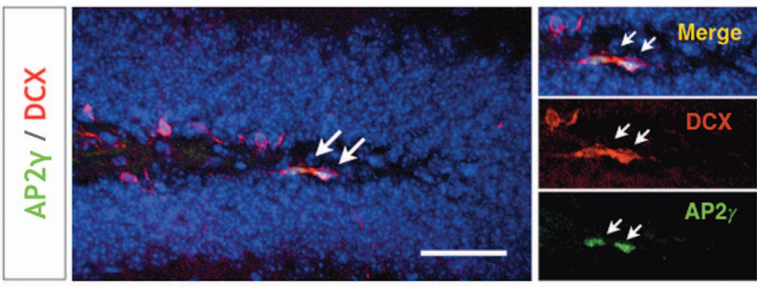

d

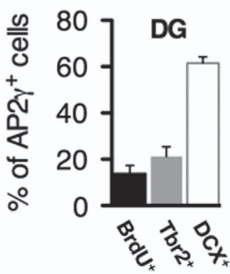

Figure 1. Activating protein $2 \gamma$ (AP2 $\gamma$ ) expression in the adult mouse hippocampal dentate gyrus (DG). (a) In situ hybrization (ISH) of AP2 $\gamma$ in the adult hippocampal DG. (b) The left panel shows the combination of ISH of AP2 $\gamma$ in the DG with immunolabelled bromodeoxyuridine (BrdU)-positive (in red) and Tbr2-positive cells (in green). (c and d) Immunohistochemical quantification of the percentage of AP2 $\gamma$-positive cells colabelled with BrdU, Tbr2 or doublecortin (DCX) in the DG. Error bars represent s.e.m. Scale bars represent $100 \mu \mathrm{m}$ (a) and $50 \mu \mathrm{m}$ (b and $\mathbf{c}$ ).

AP2 $\gamma$ regulates adult hippocampal proliferation and neuronal differentiation, through reciprocal interactions with transcriptional regulators of glutamatergic neurogenesis

After identifying the presence of AP2 $\gamma$ in glutamatergic progenitors and neuroblasts of the adult DG, we assessed whether its ability to regulate neurogenesis during the prenatal cortical developmental window was preserved in the adult brain. To understand its role in neuronal fate specification, we used NSCs primary cultures, derived from the adult DG. We used a retroviralbased approach to infect cultured NSCs from mice containing AP2 $\gamma$ flanked by loxP sites (AP2 $\gamma f l / f l$ mice) to delete AP2 $\gamma$. Viralmediated deletion of AP2 $\gamma$ produced a decrease in the generation of mixed clones (clones containing both neuronal Tuj1-positive and non-neuronal Tuj1-negative cells; $t_{18}=5.705, P<0.001$ ), counterbalanced by a marked increase in the formation of nonneuronal Tuj1-negative clones $\left(t_{18}=7.173, P<0.001\right)$, supporting the role of $A P 2 \gamma$ in commitment and differentiation into the neuronal lineage (Figures $2 a$ and b). We did not observe a significant difference in the clone size of control and AP $2 \gamma$-absent cells (Figure $2 \mathrm{c}$ ).

To verify if the effects observed in vitro upon deletion of AP $2 \gamma$ were present in the adult brain, we used tamoxifen-inducible AP2Yfl/fl//Glast::CreErt2//Z/EG mice (henceforth referred to as $\mathrm{AP} 2 \mathrm{\gamma}^{-1-}$ ) to promote the deletion of $\mathrm{AP} 2 \gamma$, and evaluated the 
effects on hippocampal neurogenesis 1 week after induction (cells with AP2 $\gamma$ deletion become labeled as GFP-positive cells). In $\mathrm{AP} 2 \mathrm{\gamma}^{-1-}$ mice, we observed a significant decrease in the percentage of GFP/DCX-double-positive cells in the DG in comparison with Wt mice $\left(t_{18}=4.239, P<0.001\right)$ (Figure $2 d$ ).
The decrease in neuroblasts was accompanied by an increase in GFP/GFAP-double-positive cells $\left(t_{18}=4.171, P<0.001\right.$; Figure $2 \mathrm{~d}$ ). This increase in GFAP-positive cells in the SGZ is likely to represent an increase in the GFAP-expressing progenitors pool, as a result of a defect in differentiation progression into glutamatergic neurons.

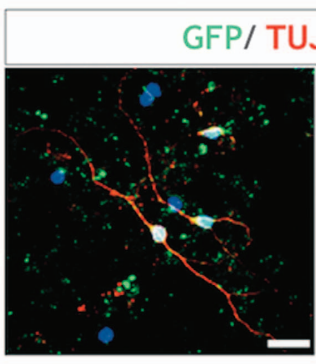

C

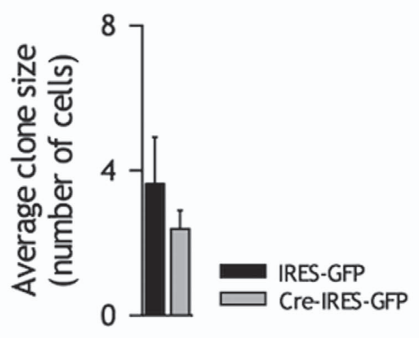

d

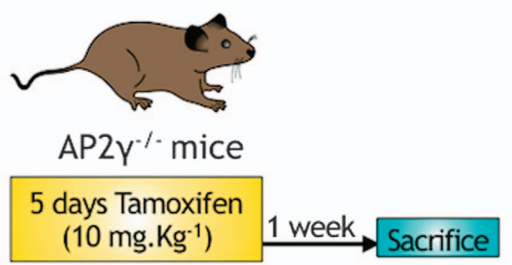

b

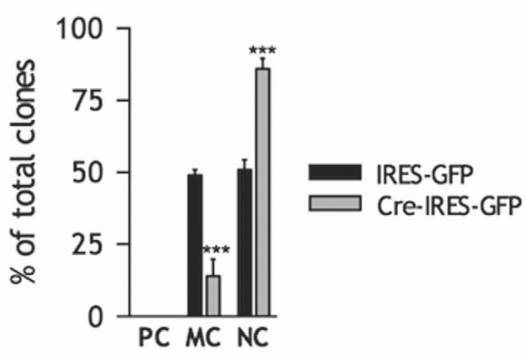

e

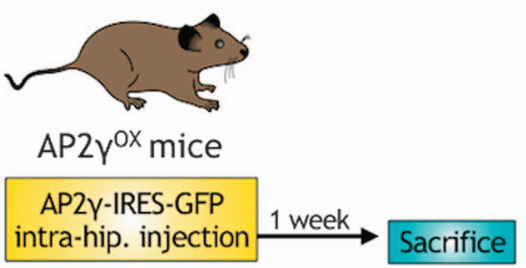

f

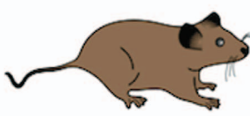

AP $2 \gamma^{0 \mathrm{X}}$ mice

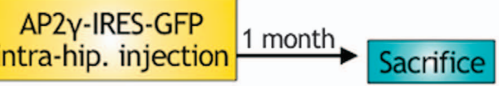

g

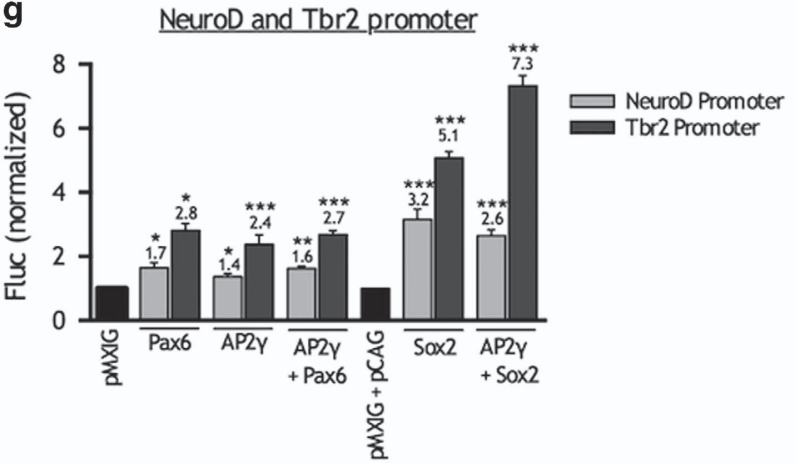

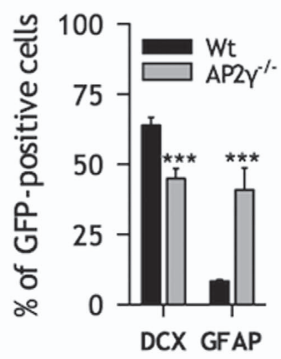
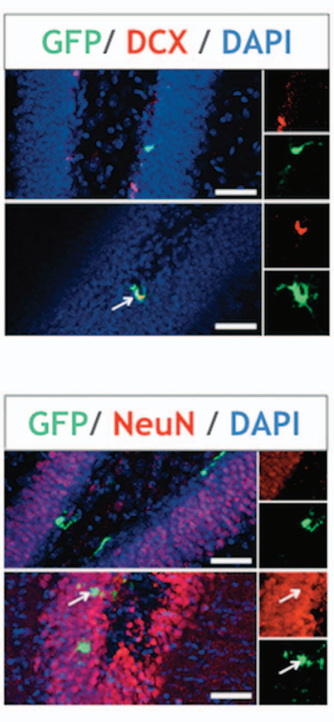

h

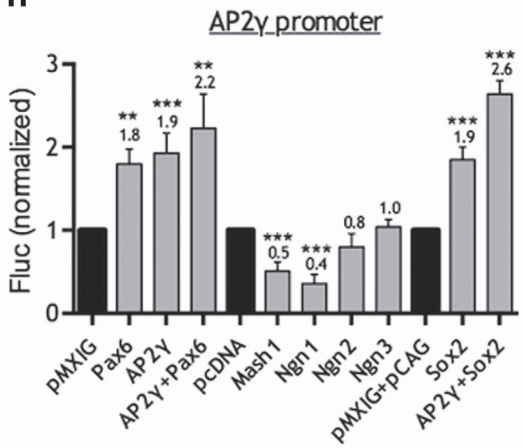


We complemented these data with a forebrain AP2 $\gamma$ deletion experiment, and observed a decrease in DCX-positive neuroblasts in the DG (Supplementary Figure 2).

To gain further insight on the effects of AP2 $\gamma$ in the regulation of adult hippocampal neurogenesis, an AP2 $\gamma$ overexpression $\left(\mathrm{AP} 2 \gamma^{\mathrm{ox}}\right.$ ) experiment was conducted through intrahippocampal injections of a retrovirus carrying an AP $2 \gamma$-IRES-GFP cassette in the DG. Hence, proliferative cells were stably infected by viral vectors, resulting in the overexpression of AP2 $\gamma$ and coexpression of GFP. Analysis performed 1 week after injection showed that a large proportion of GFP-positive cells corresponded to neuroblasts (GFP/DCX-double-positive cells; Figure 2e). Moreover, there was a reduction in the percentage of neuroblasts in $\mathrm{AP} 2 \mathrm{\gamma}^{\mathrm{ox}}$ animals, 1 week after injection $\left(t_{18}=3.082 ; P=0.003\right)$ (Figure $2 \mathrm{e}$ ) that was accompanied by a significant increase in mature granular neurons (GFP/NeuN-double-positive cells; $t_{18}=4.945 ; P<0.001$ ) (Figure $2 \mathrm{e}$ ) and a reduction in the GFAP-positive cell population $\left(t_{18}=2.828\right.$; $P=0.006$ ) (Figure 2e). This result suggests the promotion of neurogenesis and an acceleration of the neuronal differentiation process after AP2 $\gamma$ overexpression. In animals killed 1 month after injection, most GFP-positive cells corresponded to mature (NeuNpositive) neurons (Figure $2 \mathrm{f}$ ). At this time point, the increase in the differentiation of neuronal cells in the DG was maintained in $\mathrm{AP} 2 \gamma^{\mathrm{ox}}$ mice, which presented a significant increase in the percentage of GFP/NeuN-double-positive cells $\left(t_{18}=6.529 ; P<0.001\right)$ (Figure 2f). Few GFP-positive cells colocalized with GFAP-positive cells in the DG (Supplementary Figure 3) and a significant increase was observed in the percentage of GFP/GFAP-double-positive cells in AP2 $\gamma^{\text {ox }}$ mice (Figure 2f). Taken together, both in vitro and in vivo results demonstrate the role of $A P 2 \gamma$ in the regulation of adult hippocampal proliferation and neuronal differentiation.

Moreover, to have a mechanistic view on how AP $2 \gamma$ participates in the regulation of adult hippocampal neurogenesis, mouse embryonic carcinoma P19 cells were transfected with a pGL3 luciferase vector containing either NeuroD or Tbr2 promoters along with a control, Sox2, Pax6 or AP2 $\gamma$ cDNA expression vector. Transfection with AP2 $\gamma$ led to a significant activation of both promoters (Tbr2, $P<0.001$; NeuroD, $P=0.031$; Figure 2g) in line with previous reports focusing developmental stages. ${ }^{23}$ We also observed significant activation of these promoters by Sox2 (Tbr2, $P=0.001$; NeuroD, $P=0.001$; Figure $2 \mathrm{~g}$ ) and Pax6 (Tbr2, $P=0.026$; NeuroD, $P=0.022$; Figure $2 \mathrm{~g}$ ), but no cooperative effects of Pax6 with AP2 $\gamma$. However, simultaneous transfection with Sox 2 and AP2 $\gamma$ potentiated the activation of the Tbr2 promoter (Tbr2, $P=0.026$; NeuroD, $P=0.022$; Figure $2 \mathrm{~g}$ ). In a parallel assay, we also found that Pax6 and Sox2 activate the AP2 $\gamma$ promoter while Mash1 and Ngn1 promote its inhibition. Finally, we show that $\mathrm{AP} 2 \gamma$ was able to trigger its own promoter activation (Figure $2 \mathrm{~h}$ ).

AP2 $\gamma$ cKO adult mice display hippocampal neurogenesis impairments but no alterations in neuronal morphology

After using complementary approaches to manipulate AP2 $\gamma$ levels both in vitro and in vivo, a tamoxifen-inducible AP $2 \gamma$ cKO mouse model was used to explore the functional implications of AP2 $\gamma$ deficiency in the adult brain (Figure 3a). A significant reduction on AP2 $\gamma$ protein levels was confirmed by western blot in the hippocampal region of both heterozygous (AP2 $\left.\gamma^{+/-} \mathrm{CKO}\right)$ and homozygous conditional knockout (AP2 $\gamma^{-1-}$ CKO) mice (Figures $3 \mathrm{~b}$ and $\mathrm{c}$ ). Moreover, AP2 $\gamma$ deficiency triggered a reduction of Pax6 and Tbr2 protein levels, but not Sox2 (an upstream regulator), in the both dorsal and ventral DG of adult mice (Figures $3 \mathrm{~b}$ and $\mathrm{c}$ ).

AP2 $\gamma$ CKO animals present deficits in hippocampal proliferation (decrease in BrdU-positive cells), an effect that is more pronounced in homozygous mice (dorsal DG: $F_{3-37}=11.97$, $P<0.001$; post hoc: $P<0.001$; ventral $D G: F_{3-37}=8.596$, $P<0.001$; post hoc: $P<0.001$; Figures $3 \mathrm{~d}-\mathrm{f}$ ) as well as in the generation of new neuroblasts (detected as lower number of BrdU/DCX-double-positive cells; dorsal DG: $F_{3-37}=19.87, P<0.001$; post hoc: $P<0.001$; ventral DG: $F_{3-37}=4.678, P=0.015$; post hoc: $P<0.01$; Figures $3 e$ and $f$ ).

To explore whether AP2 $\gamma$ deficiency could affect other forms of structural plasticity within the adult DG, we analyzed the dendritic morphology of DG granular neurons, and spine densities and morphology (Figures $3 g-i$ and Supplementary Figure 4). Of note, none of these parameters was affected by AP2 $\gamma$ deletion.

AP2 $\gamma$ deficiency induces cognitive deficits, but has no impact on anxiety- or depressive-like behavior

Given the role of AP2 $\gamma$ in adult hippocampal neurogenesis, we tested AP2 $\gamma$ cKO mice in different behavioral paradigms to assess its impact in several emotional and cognitive domains. We used two behavioral tests to detect anxiety-like behavior, namely the open-field test and the elevated plus maze. AP2 $\gamma$ deletion was not sufficient to produce a statistically significant decrease in the total distance traveled in the center of the open-field arena (Figure 3j), or a decreased exploration time in open arms of the elevated plus maze (Figure 3k). Moreover, in the FST, a depressive-like behavior test, AP2 $\gamma$ cKO mice displayed similar immobility levels compared with $\mathrm{Wt}$ animals (Figure $3 \mathrm{l}$ ).

Next, we assessed the repercussions of AP2 $\gamma$ deletion for different cognitive domains. We tested animals in a contextual fear conditioning task, previously described to be sensitive to neurogenesis impairments. ${ }^{29}$ Animals were submitted to a context probe, aimed to test hippocampal-dependent memory, and a light-cued probe, aimed to assess the integrity of extrahippocampal memory circuits $^{29}$ (Figure $3 \mathrm{~m}$ ). All groups presented similar average freezing percentages after the conditioning trials (Figure $3 \mathrm{~m}$ ). In the context probe (context $\mathrm{A}$ ), AP $2 \mathrm{\gamma}^{-\prime-} \mathrm{CKO}$ presented a reduction in the percentage of freezing when exposed to a familiar context $\left(F_{3-15}=3.767, P=0.047\right.$; post hoc: $P<0.05$; Figure $3 \mathrm{~m}$ ). Switching to a new environment (context $B$ ) promoted a decrease in freezing in all groups (Figure $3 \mathrm{~m}$ ). Of note, heterozygous deletion of AP2 $\gamma$ was not sufficient to produce impairments in contextual memory. In the light probe, all groups presented similar responses to the light cue $\left(t_{14}=0.7959\right.$,

Figure 2. Deletion and overexpression of activating protein $2 \gamma(\mathrm{AP} 2 \gamma)$ in the adult brain. (a and $\mathbf{b})$ In vitro viral-mediated deletion of AP2 $\gamma$ in neural stem cell (NSC) primary cultures (a) with quantification of the percentage of pure neuronal (PC), mixed neuronal and non-neuronal (MC) and non-neuronal clones (NC) (b) and average clone size (c) in dentate gyrus (DG) NSC primary cultures; $n=10$. (d) Tamoxifen-induced AP2 $\gamma$ deletion in Glast-expressing cells was performed, and the percentage of green fluorescent protein (GFP)-positive cells colabelled with doublecortin (DCX) or glial fibrillary acidic protein (GFAP) in the subgranular zone (SGZ) was assessed. (e and f) Viral-mediated overexpression of AP2 $\gamma$ in the adult hippocampal DG. Adult mice were injected with retrovirus containing AP2 $\gamma$ IRES-GFP or simply IRES-GFP as an experimental control and killed either 1 week after injection (e) or 4 weeks after injection (f). Graphs show quantification of GFP-positive cells colabelled with DCX, neuronal nuclei (NeuN) or GFAP. Right panels show GFP-positive transfected cells in the hippocampal SGZ; $n=6$. (g and $\mathbf{h}$ ) Histograms depicting the luciferase luminescence intensity normalized to Renilla intensity from embryonic carcinoma P19 cells transduced with the firefly or Renilla luciferase constructs (Fluc or Rluc, respectively) using either NeuroD and Tbr2 (g) or AP2 $\gamma$ promoters (h). Values were normalized to the pMXIG empty vector containing only GFP (four independent experiments). Student's $t$-test, ${ }^{*} P \leqslant 0.05$, ${ }^{* *} P \leqslant 0.01$ and ${ }^{* * *} P \leqslant 0.001$. Error bars represent s.e.m. Scale bars represent $20 \mu \mathrm{m}$. 


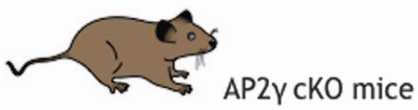

week week week week week week week week

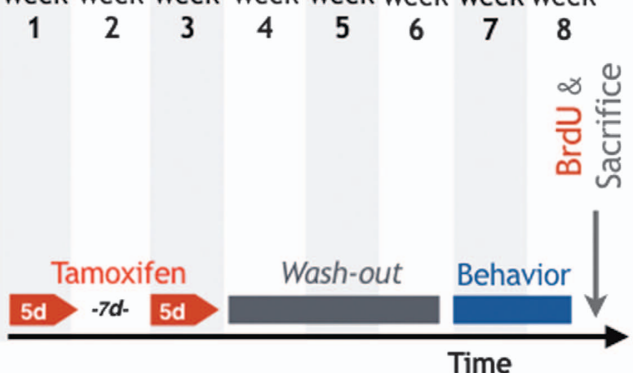

d

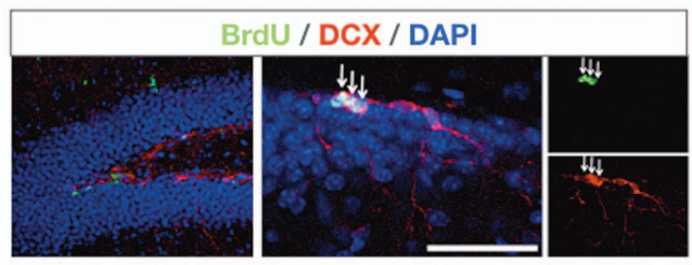

g
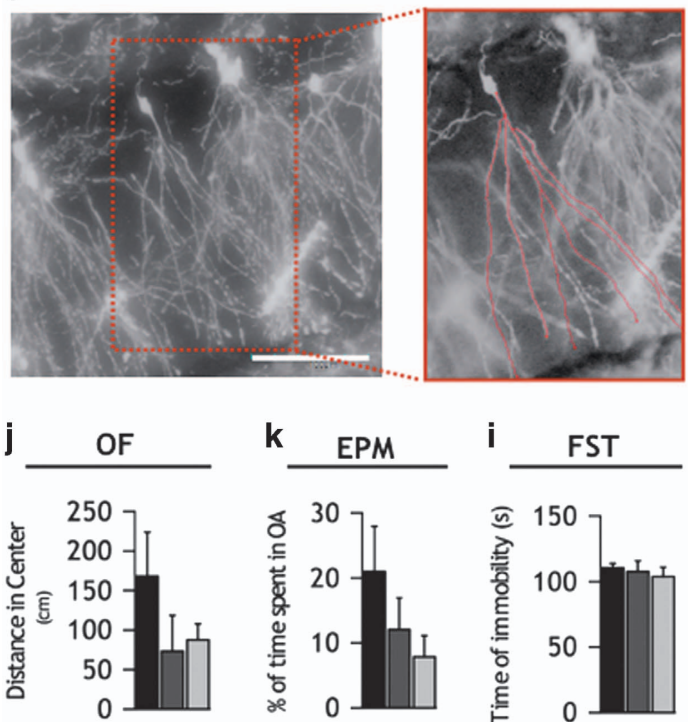
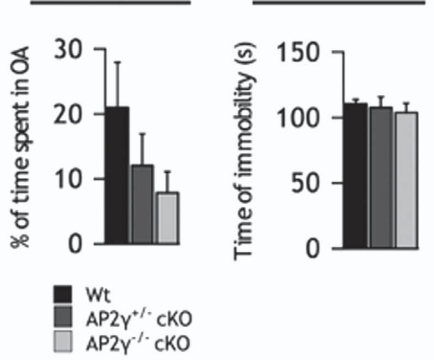

b
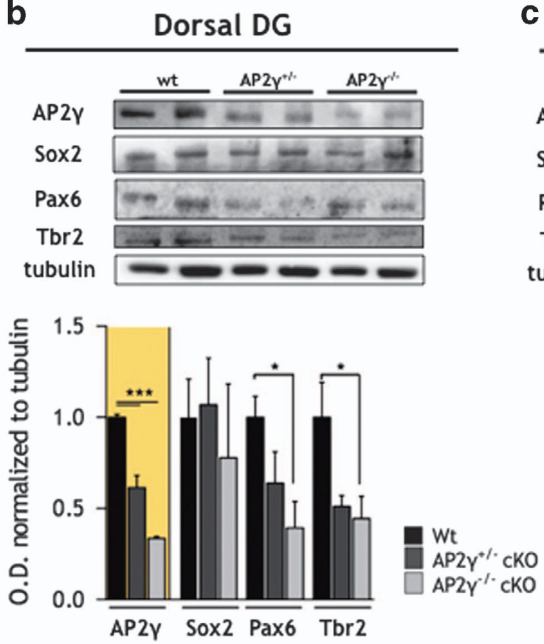

e

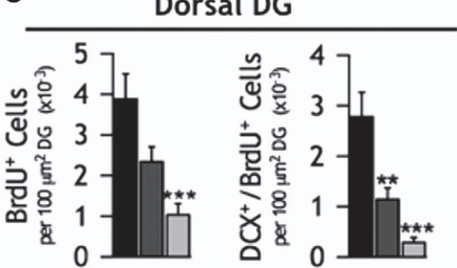

f

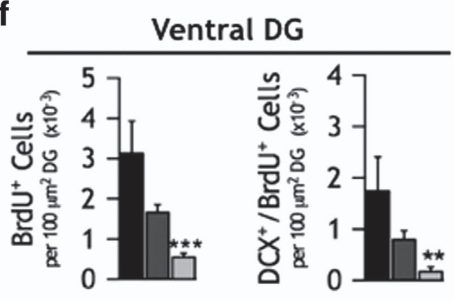

h

Dorsal DG

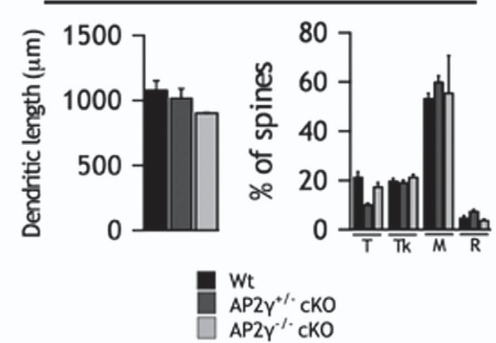

i

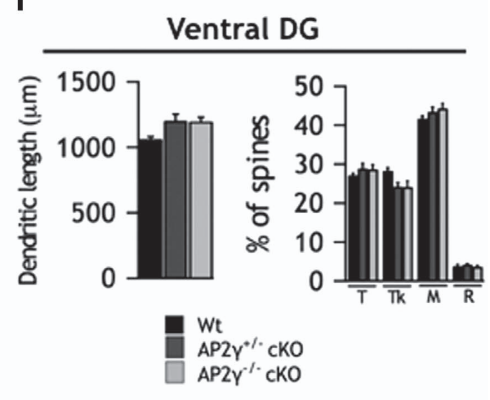

m

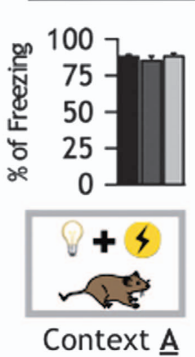

CFC
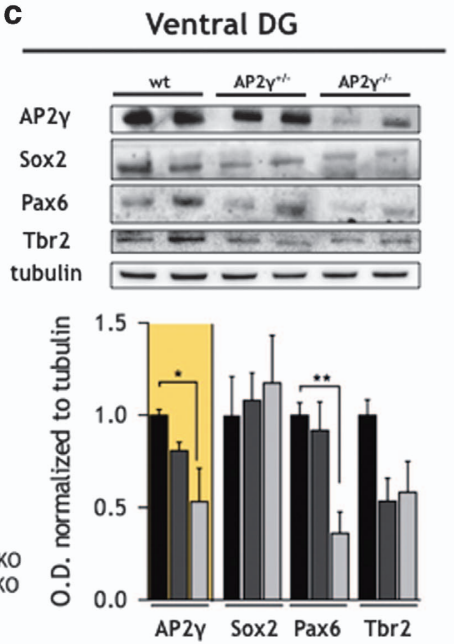

Ventral DG

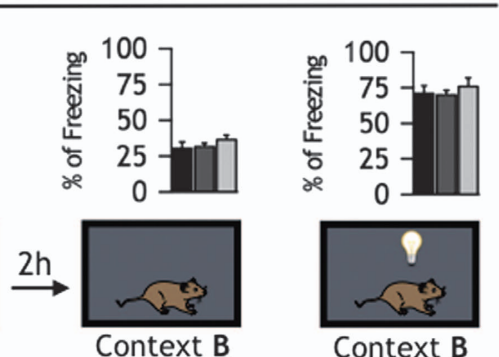

Figure 3. Loss of activating protein $2 \gamma$ (AP2 $\gamma$ ) impairs adult hippocampal neurogenesis and cognitive function. (a) Two-month-old wild-type (Wt), AP2 $\gamma f l /+/ / G l a s t:: C r e E r t 2\left(A P 2 \gamma^{+/-}\right.$CKO) and AP2 $\gamma f l / f l / / G l a s t:: C r e E r t 2 ~\left(A P 2 \gamma^{-/-}\right.$CKO) animals were injected with tamoxifen, tested 21 days after and subsequently killed. (b and $\mathbf{c}$ ) Western blot analysis of AP2 $\gamma$, Sox2, Pax6 and Tbr2 in adult hippocampal protein extracts from Wt, AP $2 \gamma^{+/-}$CKO and AP $2 \gamma^{-1-}$ CKO mice; $n=5-6$. (d) Dorsal hippocampal coronal section stained for bromodeoxyuridine (BrdU) (in green) and doublecortin (DCX) (in red). Double-stained BrdU and DCX are indicated by white arrows. (e and f) Cell counts of BrdU-positive cells and BrdU/ DCX-double-positive cells in the hippocampal dentate gyrus (DG); $n=6$. (g) Representative three-dimensional (3D) morphometric reconstruction of a DG granular neuron. (h and i) Dendritic length and spines density and morphology of hippocampal granular neurons; $n=10$. ( $\mathbf{j}$ and $\mathbf{k}$ ) Anxiety-like behavior was tested both in the open-field test (j) and in the elevated plus maze (k). (I) The presence of depressive-like behavior was assessed in the forced swim test. $(\mathbf{m})$ In addition, animals were tested in a contextual fear conditioning paradigm; percentage of freezing is presented after initial light-shock pairings (left panel), in the context probe (middle-right and -left panels) and in the cue probe (right panel); $n=10$. One-way analysis of variance (ANOVA), ${ }^{*} P \leqslant 0.05,{ }^{* *} P \leqslant 0.01$ and ${ }^{* * *} P \leqslant 0.001$. Error bars represent s.e.m. Scale bars represent $50 \mu \mathrm{m}$. CFC, contextual fear conditioning; CKO, conditional knockout; EPM, elevated plus maze; FST, forced swim test; M, mushroom spines; OA, open arms; OF, open field; R, ramified spines; $T$, thin spines; Tk, thick spines. 
a

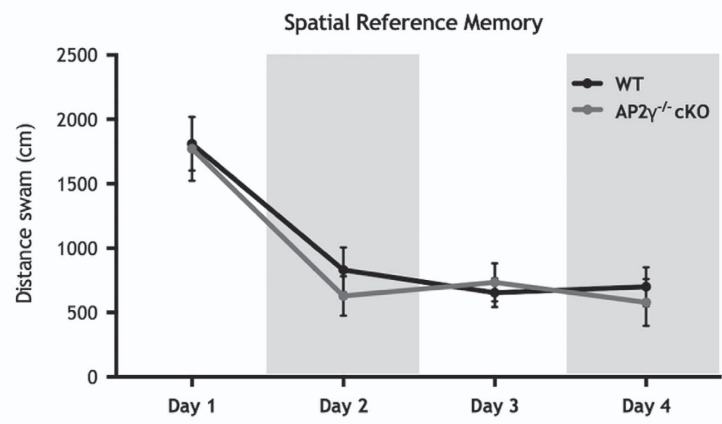

b

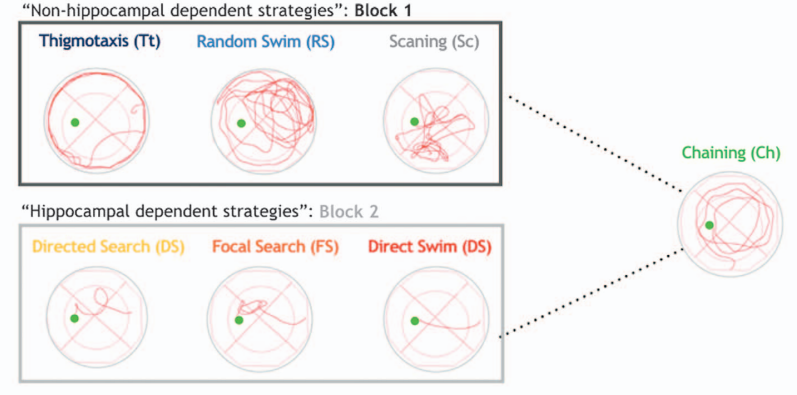

c

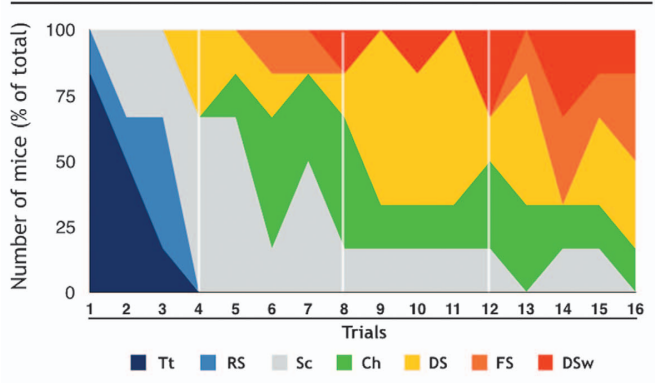

e

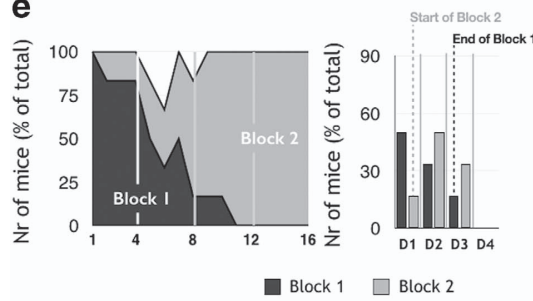

d
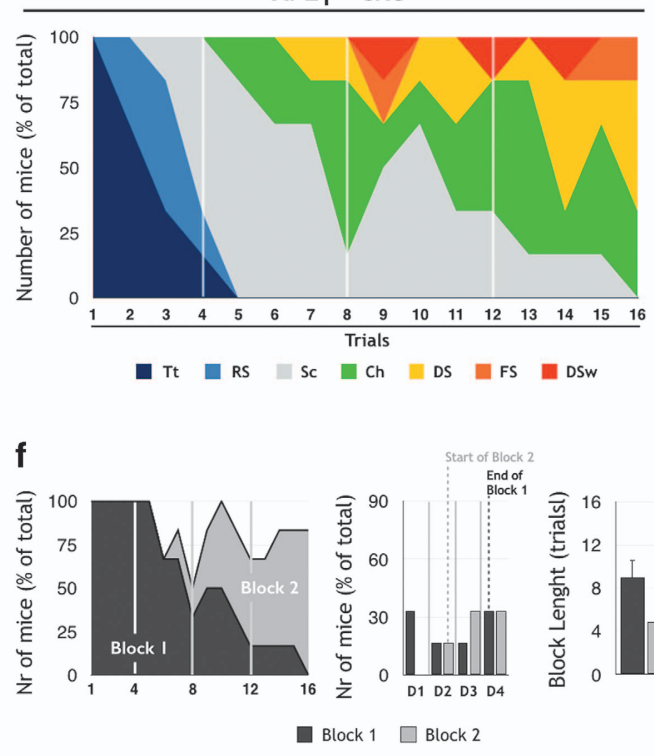

g Overlap

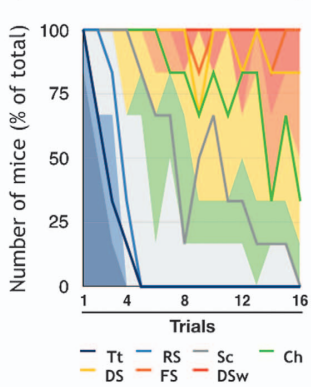

h

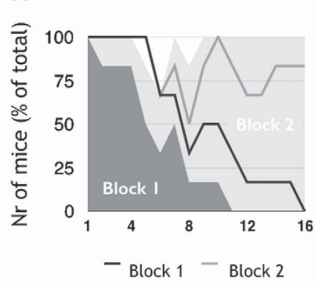

i

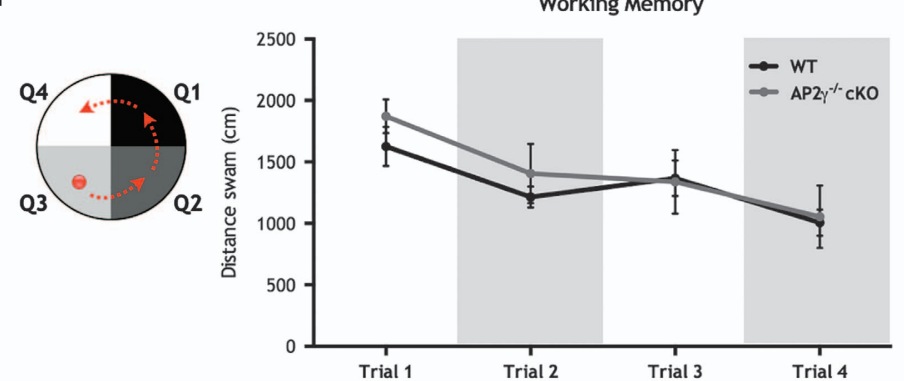

j

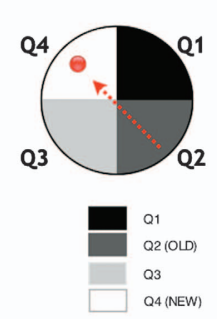

Reversal Learning

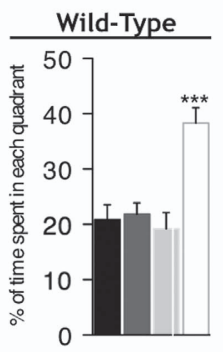

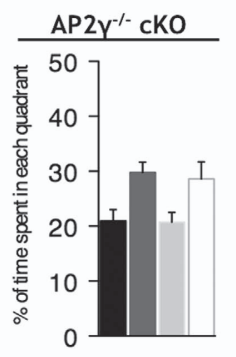

Figure 4. Cognitive strategies during water maze learning in AP2 $\gamma^{-/-}$conditional knockout (cKO) mice. (a-h) Spatial reference memory was evaluated as the average escape latency in each test day. A schematic representation and color code for each strategy (b) and the average prevalence of each strategy by trial number are shown both for wild-type $(\mathrm{Wt})(\mathbf{c})$ and for AP2 $2 \gamma^{-/-} \mathrm{cKO}$ animals (d). The prevalence of each strategy-block along trials (Block 1: 'non-hippocampal-dependent strategies'; Block 2: 'hippocampal-dependent strategies'), the distribution of strategies-block boundaries and overall block length are shown for Wt (e) and AP $2 \gamma^{-1-}$ cKO animals (f); graphical comparison of these parameters is shown in ( $\mathbf{g}$ and $\mathbf{h})$. (i and $\mathbf{j})$ Furthermore, animals were tested in a working memory task (i) and in reversal learning task (j); $n=10$, Student's $t$-test, ${ }^{* * *} P \leqslant 0.001$. Error bars represent s.e.m. AP $2 \gamma$, activating protein $2 \gamma$.

$P=0.219$; Figure $3 \mathrm{~m})$. Overall, contextual fear conditioning results showed that $\mathrm{AP} 2 \mathrm{\gamma}^{-/-} \mathrm{CKO}$ display specific deficits in contextual hippocampal-associated memory, whereas preserving associative non-hippocampal-dependent memory.

We proceeded with the cognitive characterization of AP2 $\gamma$ cKO using different water maze test paradigms (Figure 4). In a reference memory task, which relies on the integrity of hippocampal function, ${ }^{30} \mathrm{Wt}$ and $\mathrm{AP} 2 \gamma^{-1-}$ CKO mice presented similar learning curves (Figure 4a). However, analysis of the strategies adopted to reach the escape platform ${ }^{31-33}$ showed that
$\mathrm{AP} 2 \mathrm{\gamma}^{-1-}$ cKO mice delayed the switch from non-hippocampaldependent strategies ('Block $1^{\prime}$ ) to hippocampal-dependent strategies ('Block 2') (Figures 4b-h). In fact, most AP $2 \gamma^{-/-}$cKO animals initiate Block 2 strategies by test days 3 and 4 , while presenting an increased mean duration of Block 1 compared with Wt mice (Block 1: $t_{18}=1.966 ; P=0.032 ;$ Block 2: $t_{18}=2.690$; $P=0.008$; Figures $4 \mathrm{e}-\mathrm{h}$ ). Furthermore, in a working memory test paradigm, $\mathrm{AP} 2 \mathrm{\gamma}^{-1-} \mathrm{CKO}$ and Wt mice presented similar performances along all the trials (Figure 4i). Regarding behavioral flexibility, AP $2 \mathrm{\gamma}^{-1-}$ CKO displayed increased time spent on the 
a

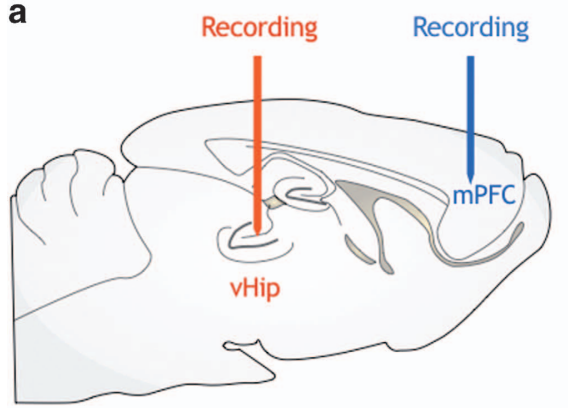

b

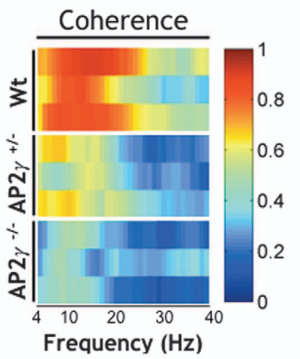

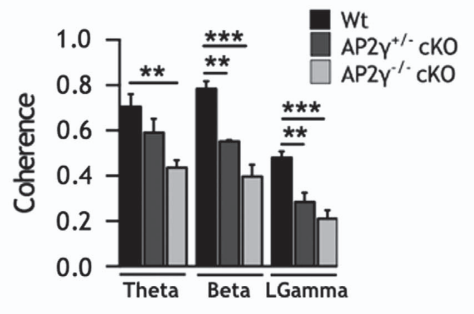

C

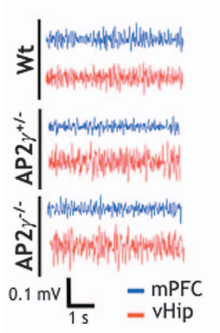

d PSD vHip

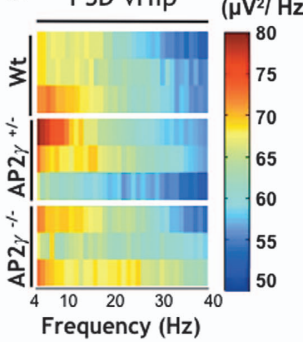

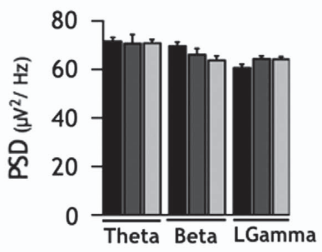
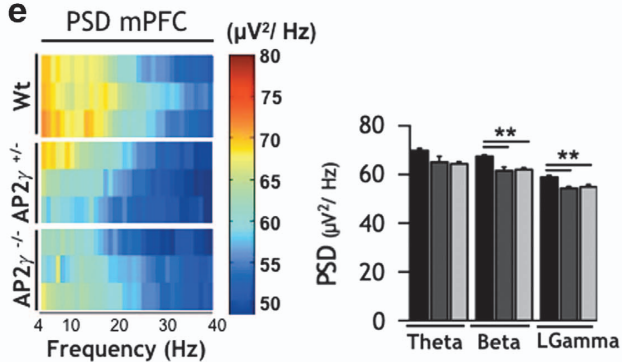

Figure 5. Activating protein $2 \gamma(\mathrm{AP} 2 \gamma)$ deficiency decreases spectral coherence between the ventral hippocampus (vHIP) and the medial prefrontal cortex (mPFC) and neuronal activity within each region. (a) Upper panel depicting local field potential (LFP) recording sites and electrode positions; lower panel shows representative LFP signals. (b) Spectral coherence between vHIP and mPFC of wild-type (Wt) and AP2 $\gamma$ conditional knockout (cKO) mice (left panel); group comparison of the coherence values for each frequency band (right panel). (c) Representative traces of power spectral density (PSD) raw data recorded simultaneously from the mPFC (blue line) and the vHIP (red line) of a rat of each group. (d) PSD measured in the vHIP (left panel); group comparison of the PSD values for each frequency band (right panel). (e) PSD measured in the mPFC (left panel); group comparison of the PSD values for each frequency band (right panel). In the spectrograms each horizontal line in the $Y$ axis represents the spectrogram of an individual mouse (three representative mice from each group are shown); $n=5$. One-way analysis of variance (ANOVA), ${ }^{* *} P \leqslant 0.01$ and ${ }^{* * *} P \leqslant 0.001$. Error bars represent s.e.m.

'older quadrant', compared with Wt mice $\left(t_{18}=4.806, P<0.0001\right.$; Figure 4j). Interestingly, these results were only found in homozygous mice, as AP2 $\gamma^{+/-}$cKO presented test performances similar to Wt animals (Supplementary Figure 5).

The hippocampal-to-PFC link is impaired in AP2 $\gamma^{-1-}$ CKO mice To better understand the mechanism underlying reference memory and behavior flexibility deficits in AP2 $\gamma$-deficient mice, we performed a functional characterization of the hippocampusto-PFC network by analyzing electrophysiological features of LFPs in these areas (Figure 5a). Interestingly, the temporal structure of the LFPs recorded from AP2 $\gamma$ cKO animals was found to be affected; specifically, in AP $2 \gamma^{-1-} \mathrm{CKO}$ animals, coherence measurements between simultaneously recorded LFPs ${ }^{28,34}$ of the medial PFC and the vHIP were significantly decreased in all spectral bands (theta: $F_{3-12}=6.788, P=0.011$, post hoc: $P<0.01$; beta: $F_{3-12}=28.72$, $P<0.001$, post hoc: $P<0.001$; low gamma: $F_{3-12}=19.77, P<0.001$, post hoc: $P<0.001$; Figure $5 \mathrm{~b}$ ), thus showing a compromised connection between these two brain regions. PSDs translate the amplitude of the signals recorded in a brain region across the frequency domain and are important read-outs of the function of that region. ${ }^{28,35}$ AP2 $\gamma$ depletion did not exert an effect in PSD of the ventral hippocampus (Figures $5 \mathrm{c}$ and $\mathrm{d}$ ) but promoted a reduction in PSD of the PFC, namely in the beta and low gamma frequency bands (beta: $F_{3-12}=11.94, P=0.001$, post hoc: $P<0.01$; low gamma: $F_{3-12}=11.03, P=0.0038$, post hoc: $P<0.01$; Figures $5 \mathrm{c}$ and $\left.\mathrm{e}\right)$.

\section{DISCUSSION}

Adult hippocampal neurogenesis has been widely associated with hippocampal-dependent cognitive functions, such as spatial reference memory, behavioral flexibility or pattern separation. ${ }^{36-38}$
In addition, and although still a matter of debate, altered hippocampal neurogenesis has been implicated in the precipitation of anxiety- and depressive-like behavior in rodent models of psychiatric diseases, as well as in the improving effects mediated by different classes of antidepressants, antipsychotic or antidementia drugs. ${ }^{10,39,40}$ Herein, we investigated whether the neurogenic regulatory effects of AP2 $\gamma$ in the developing brain could be extended to the mature adult brain.

We believe the present study demonstrates for the first time the presence of AP2 $\gamma$ in the adult hippocampal DG, both in Tbr2positive glutamatergic progenitor cells and in neuroblasts. Moreover, results reveal that AP2 $\gamma$ is a positive regulator of adult hippocampal neurogenesis. Its overexpression promotes the generation of new neurons in this region, whereas its deletion results in a marked reduction of the neuroblast population, both in vitro and in vivo. Mechanistically, AP2 $\gamma$ acts as an effector of Sox2 and Pax6 in the promotion of Tbr2 expression in hippocampal progenitor cells. In fact, we show that alterations in AP2 $\gamma$ expression produce a negative net effect in Tbr2 protein levels within the hippocampal DG (significant decrease). The results suggest that $\mathrm{AP} 2 \gamma$ regulates postnatal glutamatergic neurogenesis by mobilizing TAPs, rather than interfering with the NSC pool. Indeed, Tbr2 (along with transcription factors such as NeuroD) is likely a major downstream effector of AP2 $\gamma$ regulation. Tbr2 expression has been shown to be critical for TAPs' pool expansion and to coordinate the progression to subsequent neuronal lineage differentiation stages in the adult hippocampus. ${ }^{22,41,42}$ Interestingly, the presence of an alternative regulatory pathway using $\mathrm{AP} 2 \gamma$ as an intermediate transcriptional regulator, in parallel with the direct regulation of Tbr2 by Pax6, suggests that AP2 $\gamma$ function may allow a fine-tuning of the neurogenic process. This may be either by rapidly expanding or by restricting the TAPs' pool through the modulation of Tbr2 
expression. ${ }^{43,44}$ Accordingly, the reduction of progenitor cells observed after deletion of AP2 $\gamma$ possibly results from a failure in the progression to a postmitotic phase, where normal axonal growth and dendritic extension allow the proper synaptic input (shown to be critical for the successful survival and maturation of newborn cells). ${ }^{1,6}$ More so, AP $2 \gamma$ deletion in early embryonic corticogenesis was associated with a twofold increase in apoptosis of progenitor cells and their immediate progeny. ${ }^{23}$ Thus, it is plausible that the same developmental outcome is recapitulated in adult hippocampus and the observed reduction in TAPs is related with halted progression to subsequent maturation stages, culminating in cell death of glutamatergic progenitors.

We next explored how the transcriptional modulation of glutamatergic neurogenesis could impact on behavior. Interestingly, no significant changes were observed in emotional states, both depressive- or anxiety-like, in animals with reduced levels of AP2 $\gamma$. Given that AP2 $\gamma$ is only present in a subset of newly formed neuroblasts, it is likely that the lack of AP2 $\gamma$-positive neuroblast sub-population is not sufficient to elicit an evident phenotype. Moreover, AP2 $\gamma$ manipulation in the adult hippocampus did not influence normal dendritic morphology of postmitotic cells, another form of hippocampal structural plasticity critical for complex emotional behaviors. ${ }^{7,40}$ Altogether, results point for the need to characterize and modulate AP2 $\gamma$-positive and -negative neuroblast populations in future studies. This will allow to pinpoint its specific participation in different behavioral outcomes, both in basal and in pathological context. Furthermore, it is plausible that by challenging the finely tuned hippocampal neurogenic process, AP2 $\gamma$-positive newborn cells will evidence additional functional correlates. Accordingly, glutamatergic Tbr2-positive progenitors have been shown to be highly responsive to environmental enrichment or voluntary wheel running, which more than doubled Tbr2-positive TAPs, suggesting that in the advent of external stimuli these cells may have additional roles to those here reported. Additional insights on the full extent of the functional importance of AP2 $\gamma$-positive progenitors may come from future studies analyzing the behavioral impacts of AP2 $\gamma$ overexpression in the adult hippocampus. More so, in studies, in which hippocampal neurogenesis has been experimentally bolstered, beneficial effects in learning, memory ${ }^{45,46}$ and pattern separation ${ }^{47}$ were reported. In the opposite perspective, in psychopathological contexts known to promote a potent antineurogenic insult, such as chronic stress exposure, the sub-population of AP2 $\gamma$-positive progenitors is likely to become severely compromised. This reduction in AP $2 \gamma$-positive cells, in articulation with other deleterious effects on neural plasticity promoted by chronic stress, may also contribute to a better characterization of the importance of these cells, not only in basal conditions but also in pathological scenarios, such as in depression. ${ }^{9,48}$

Interestingly, AP2 $\gamma$ regulation of the TAPs' population seems essential to the preservation of hippocampal-dependent cognitive tasks. Cognitive dimensions based on the interaction of the hippocampal formation and prefrontal cortical areas, such as spatial behavioral flexibility, were also impaired in AP $2 \gamma^{-1-} \mathrm{CKO}$ animals. Strikingly, the electrophysiological studies revealed that AP2 $\gamma$ deficiency in the adult brain led to a significant decrease of coherence between the VHIP and the PFC, indicating a decrease in the ability of these regions to functionally interact. This included the $\theta$ and $\beta$ frequencies, previously shown to be critically related with behavior outputs dependent on the corticolimbic networks. ${ }^{28,49,50}$ Such inter-regional electrophysiological impairments reflect how the lack of AP2 $\gamma$-positive progenitors impact not only at the level of intrahippocampal circuitry but also modulate the function of cortical regions that cooperate with the hippocampus in the orchestration of complex cognitive behaviors. Moreover, the integrity of the vHIP-to-PFC link has been recently described to be important to the antidepressant action of drugs, such as ketamine, ${ }^{51}$ raising the possibility of $A P 2 \gamma$ to have an important role in the preservation of this neuronal circuit.

Altogether, in this work we show that the lack of AP2 $\gamma$ in the adult mammalian brain impairs the regulation of hippocampal neurogenesis, leading to glutamatergic network malfunction impairments on neuronal activity and inter-regional communication. This dysregulation had significant implications for cognitive processes that may be relevant for the pathogenesis of psychiatric conditions. In light of the findings reported herein, future studies should explore whether AP2 $\gamma$ participates in the pathogenesis of these disorders characterized by hippocampal neurogenesis impairments.

\section{CONFLICT OF INTEREST}

The authors declare no conflict of interest.

\section{ACKNOWLEDGMENTS}

We acknowledge the excellent technical expertise of Luís Martins and Andrea Steiner-Mezzadri. We would also like to acknowledge Magdalena Götz for the insightful comments on the paper. AMP, PP, ARS, JS, VMS, NDA and JFO received fellowships from the Portuguese Foundation for Science and Technology (FCT). LP received fellowship from FCT and her work is funded by FCT (IF/01079/2014) and Bial Foundation (427/14) projects. This work was cofunded by the Life and Health Sciences Research Institute (ICVS), and Northern Portugal Regional Operational Programme (NORTE 2020), under the Portugal 2020 Partnership Agreement, through the European Regional Development Fund (FEDER) (projects NORTE-01-0145FEDER-000013 and NORTE-01-0145-FEDER-000023). This work has been also funded by FEDER funds, through the Competitiveness Factors Operational Programme (COMPETE), and by National funds, through the FCT, under the scope of the project POCI-01-0145-FEDER-007038.

\section{REFERENCES}

1 Ming GL, Song $\mathrm{H}$. Adult neurogenesis in the mammalian brain: significant answers and significant questions. Neuron 2011; 70: 687-702.

2 Alvarez-Buylla A, Garcia-Verdugo JM, Tramontin AD. A unified hypothesis on the lineage of neural stem cells. Nat Rev Neurosci 2001; 2: 287-293.

3 Zhao C, Teng EM, Summers RG Jr, Ming GL, Gage FH. Distinct morphological stages of dentate granule neuron maturation in the adult mouse hippocampus. J Neurosci 2006; 26: 3-11.

4 Merkle FT, Mirzadeh Z, Alvarez-Buylla A. Mosaic organization of neural stem cells in the adult brain. Science 2007; 317: 381-384.

5 Hodge RD, Kahoud RJ, Hevner RF. Transcriptional control of glutamatergic differentiation during adult neurogenesis. Cell Mol Life Sci 2012; 69: 2125-2134.

6 Tozuka Y, Fukuda S, Namba T, Seki T, Hisatsune T. GABAergic excitation promotes neuronal differentiation in adult hippocampal progenitor cells. Neuron 2005; 47: 803-815.

7 Bessa JM, Ferreira D, Melo I, Marques F, Cerqueira JJ, Palha JA et al. The mood-improving actions of antidepressants do not depend on neurogenesis but are associated with neuronal remodeling. Mol Psychiatry 2009; 14: 764-773, 739.

8 Kim JY, Liu CY, Zhang F, Duan X, Wen Z, Song J et al. Interplay between DISC1 and GABA signaling regulates neurogenesis in mice and risk for schizophrenia. Cell 2012; 148: 1051-1064

9 Mateus-Pinheiro A, Pinto L, Bessa JM, Morais M, Alves ND, Monteiro S et al. Sustained remission from depressive-like behavior depends on hippocampal neurogenesis. Transl Psychiatry 2013; 3: e210.

10 Akers KG, Martinez-Canabal A, Restivo L, Yiu AP, De Cristofaro A, Hsiang HL et al. Hippocampal neurogenesis regulates forgetting during adulthood and infancy. Science 2014; 344: 598-602.

11 Wu MV, Sahay A, Duman RS, Hen R. Functional differentiation of adult-born neurons along the septotemporal axis of the dentate gyrus. Cold Spring Harb Perspect Biol 2015; 7: a018978.

12 Bertrand N, Castro DS, Guillemot F. Proneural genes and the specification of neural cell types. Nat Rev Neurosci 2002; 3: 517-530.

13 Englund C, Fink A, Lau C, Pham D, Daza RA, Bulfone A et al. Pax6, Tbr2 and Tbr1 are expressed sequentially by radial glia, intermediate progenitor cells, and postmitotic neurons in developing neocortex. J Neurosci 2005; 25: 247-251. 
14 Hack MA, Saghatelyan A, de Chevigny A, Pfeifer A, Ashery-Padan R, Lledo PM et al. Neuronal fate determinants of adult olfactory bulb neurogenesis. Nat Neurosci 2005; 8: 865-872.

15 Waclaw RR, Allen ZJ 2nd, Bell SM, Erdelyi F, Szabo G, Potter SS et al. The zinc finger transcription factor Sp8 regulates the generation and diversity of olfactory bulb interneurons. Neuron 2006; 49: 503-516.

16 Brill MS, Ninkovic J, Winpenny E, Hodge RD, Ozen I, Yang R et al. Adult generation of glutamatergic olfactory bulb interneurons. Nat Neurosci 2009; 12: 1524-1533.

17 Gotz M, Stoykova A, Gruss P. Pax6 controls radial glia differentiation in the cerebral cortex. Neuron 1998; 21: 1031-1044.

18 Hevner RF, Hodge RD, Daza RA, Englund C. Transcription factors in glutamatergic neurogenesis: conserved programs in neocortex, cerebellum, and adult hippocampus. Neurosci Res 2006; 55: 223-233.

19 Esposito MS, Piatti VC, Laplagne DA, Morgenstern NA, Ferrari CC, Pitossi FJ et al. Neuronal differentiation in the adult hippocampus recapitulates embryonic development. J Neurosci 2005; 25: 10074-10086.

20 Nacher J, Varea E, Blasco-Ibanez JM, Castillo-Gomez E, Crespo C, Martinez-Guijarro FJ et al. Expression of the transcription factor Pax 6 in the adult rat dentate gyrus. J Neurosci Res 2005; 81: 753-761.

21 Song H, Kempermann G, Overstreet Wadiche L, Zhao C, Schinder AF, Bischofberger J. New neurons in the adult mammalian brain: synaptogenesis and functional integration. J Neurosci 2005; 25: 10366-10368.

22 Hodge RD, Nelson BR, Kahoud RJ, Yang R, Mussar KE, Reiner SL et al. Tbr2 is essential for hippocampal lineage progression from neural stem cells to intermediate progenitors and neurons. J Neurosci 2012; 32: 6275-6287.

23 Pinto L, Drechsel D, Schmid MT, Ninkovic J, Irmler M, Brill MS et al. AP2gamma regulates basal progenitor fate in a region- and layer-specific manner in the developing cortex. Nat Neurosci 2009; 12: 1229-1237.

24 Thewes V, Orso F, Jager R, Eckert D, Schafer S, Kirfel G et al. Interference with activator protein-2 transcription factors leads to induction of apoptosis and an increase in chemo- and radiation-sensitivity in breast cancer cells. BMC Cancer 2010; 10: 192.

25 Mori T, Tanaka K, Buffo A, Wurst W, Kuhn R, Gotz M. Inducible gene deletion in astroglia and radial glia--a valuable tool for functional and lineage analysis. Glia 2006; 54: 21-34.

26 Novak A, Guo C, Yang W, Nagy A, Lobe CG. Z/EG, a double reporter mouse line that expresses enhanced green fluorescent protein upon Cre-mediated excision. Genesis 2000; 28: 147-155.

27 Brill MS, Snapyan M, Wohlfrom H, Ninkovic J, Jawerka M, Mastick GS et al. A dl×2- and pax6-dependent transcriptional code for periglomerular neuron specification in the adult olfactory bulb. J Neurosci 2008; 28: 6439-6452.

28 Oliveira JF, Dias NS, Correia M, Gama-Pereira F, Sardinha VM, Lima A et al. Chronic stress disrupts neural coherence between cortico-limbic structures. Front Neural Circuits 2013; 7: 10

29 Gu Y, Arruda-Carvalho M, Wang J, Janoschka SR, Josselyn SA, Frankland PW et al. Optical controlling reveals time-dependent roles for adult-born dentate granule cells. Nat Neurosci 2012; 15: 1700-1706.

30 Cerqueira JJ, Mailliet F, Almeida OF, Jay TM, Sousa N. The prefrontal cortex as a key target of the maladaptive response to stress. J Neurosci 2007; 27: 2781-2787.

31 Garthe A, Behr J, Kempermann G. Adult-generated hippocampal neurons allow the flexible use of spatially precise learning strategies. PLoS One 2009; 4: e5464.
32 Ruediger S, Spirig D, Donato F, Caroni P. Goal-oriented searching mediated by ventral hippocampus early in trial-and-error learning. Nat Neurosci 2012; 15: 1563-1571.

33 Garthe A, Kempermann G. An old test for new neurons: refining the Morris water maze to study the functional relevance of adult hippocampal neurogenesis. Front Neurosci 2013; 7: 63.

34 Adhikari A, Topiwala MA, Gordon JA. Synchronized activity between the ventral hippocampus and the medial prefrontal cortex during anxiety. Neuron 2010; 65 : 257-269.

35 Varela F, Lachaux JP, Rodriguez E, Martinerie J. The brainweb: phase synchronization and large-scale integration. Nat Rev Neurosci 2001; 2: 229-239.

36 Shen L, Nam HS, Song P, Moore H, Anderson SA. FoxG1 haploinsufficiency results in impaired neurogenesis in the postnatal hippocampus and contextual memory deficits. Hippocampus 2006; 16: 875-890.

37 Deng W, Saxe MD, Gallina IS, Gage FH. Adult-born hippocampal dentate granule cells undergoing maturation modulate learning and memory in the brain. J Neurosci 2009; 29: 13532-13542.

38 Jessberger S, Clark RE, Broadbent NJ, Clemenson GD Jr, Consiglio A, Lie DC et al. Dentate gyrus-specific knockdown of adult neurogenesis impairs spatial and object recognition memory in adult rats. Learn Mem 2009; 16: 147-154.

39 Kodama M, Fujioka T, Duman RS. Chronic olanzapine or fluoxetine administration increases cell proliferation in hippocampus and prefrontal cortex of adult rat. Biol Psychiatry 2004; 56: 570-580.

40 Mateus-Pinheiro A, Patricio P, Bessa JM, Sousa N, Pinto L. Cell genesis and dendritic plasticity: a neuroplastic pas de deux in the onset and remission from depression. Mol Psychiatry 2013; 18: 748-750.

41 Hodge RD, Kowalczyk TD, Wolf SA, Encinas JM, Rippey C, Enikolopov G et al. Intermediate progenitors in adult hippocampal neurogenesis: Tbr2 expression and coordinate regulation of neuronal output. J Neurosci 2008; 28: 3707-3717.

42 Urban N, Guillemot F. Neurogenesis in the embryonic and adult brain: same regulators, different roles. Front Cell Neurosci 2014; 8: 396.

43 Bonaguidi MA, Wheeler MA, Shapiro JS, Stadel RP, Sun GJ, Ming GL et al. In vivo clonal analysis reveals self-renewing and multipotent adult neural stem cell characteristics. Cell 2011; 145: 1142-1155.

44 Taylor V. Hippocampal stem cells: so they are multipotent!. J Mol Cell Biol 2011; 3: 270-272.

45 van Praag H, Christie BR, Sejnowski TJ, Gage FH. Running enhances neurogenesis, learning, and long-term potentiation in mice. Proc Natl Acad Sci USA 1999; 96: 13427-13431.

46 Deng W, Aimone JB, Gage FH. New neurons and new memories: how does adult hippocampal neurogenesis affect learning and memory? Nat Rev Neurosci 2010; 11: $339-350$.

47 Sahay A, Scobie KN, Hill AS, O'Carroll CM, Kheirbek MA, Burghardt NS et al. Increasing adult hippocampal neurogenesis is sufficient to improve pattern separation. Nature 2011; 472: 466-470.

48 Sousa N. The dynamics of the stress neuromatrix. Mol Psychiatry 2016; 21: 302-312.

49 Fell J, Axmacher $\mathrm{N}$. The role of phase synchronization in memory processes. Nat Rev Neurosci 2011; 12: 105-118.

50 Gordon JA. Oscillations and hippocampal-prefrontal synchrony. Curr Opin Neurobiol 2011; 21: 486-491.

51 Carreno FR, Donegan JJ, Boley AM, Shah A, DeGuzman M, Frazer A et al. Activation of a ventral hippocampus-medial prefrontal cortex pathway is both necessary and sufficient for an antidepressant response to ketamine. Mol Psychiatry 2016; 21: 1298-1308.

Supplementary Information accompanies the paper on the Molecular Psychiatry website (http://www.nature.com/mp) 